\title{
Comunicación
}

\section{FRECUENCIA DE NEOPLASIAS EN FELINOS EN LA CIUDAD DE LIMA DURANTE EL PERIODO 1996-2007}

\author{
Frequency of Neoplasms in Cats in the City of Lima During the Period \\ 1996-2007
}

Talía Castro D. ${ }^{1}$, Alfonso Chavera C. ${ }^{1,2}$, Rosa Perales C. ${ }^{1}$, Viviana Fernández P. ${ }^{3}$

\section{RESUMEN}

El presente estudio tuvo como objetivo determinar la frecuencia de neoplasias diagnosticadas histopatológicamente en el Laboratorio de Patología Veterinaria de la Facultad de Medicina Veterinaria de la Universidad Nacional Mayor de San Marcos entre los años 1996-2007. Se hizo un estudio retrospectivo empleándose 73 registros de neoplasias. Los animales mayores de 9 años (49.3\%) y las hembras (72.9\%) fueron los más afectados. Asimismo, las neoplasias de origen epitelial fueron las más frecuentes (53.5\%), siendo el adenocarcinoma mamario (21.1\%) y el linfoma (16.9\%) los de mayor presentación.

Palabras clave: felinos, neoplasias, histopatología, adenocarcinoma, linfoma

\section{ABSTRACT}

The study aimed to determine the frequency of neoplasmas that were histopathologically diagnosed at the Pathology Laboratory of School of Veterinary Medicine, San Marcos University in the period 1996-2007. The retrospective study included 73 cases of neoplasms. Animals older than 9 years of age (49.3\%) and females (72.9\%) were the most affected. Tumors of epithelial origin were the most common type (53.5\%), being mammary adenocarcinoma (21.1\%) and lymphoma (16.9\%) the most frequent.

Key words: cat, neoplasm, histopathology, adenocarcinoma, lymphoma

\footnotetext{
${ }^{1}$ Laboratorio de Histología, Embriología y Patología Veterinaria, ${ }^{3}$ Clínica de Animales Menores, Facultad de Medicina Veterinaria, Universidad Nacional Mayor de San Marcos, Lima

${ }^{2}$ E-mail: achavera03@gmail.com
} 
Los felinos están sujetos a la ocurrencia de neoplasias, especialmente de tipo maligno, como el linfosarcoma, el carcinoma de células escamosas y el cáncer de glándula mamaria. Es por ello, que el Médico Veterinario clínico debe estar capacitado para poder diagnosticar los procesos tumorales, $\mathrm{y}$ brindar el tratamiento necesario y la información apropiada al dueño de la mascota (Martínez y Pérez, 2007).

El avance de la ciencia en el campo de la nutrición, desarrollo de técnicas diagnósticas y de protocolos terapéuticos y aplicación de medidas preventivas en el campo de la salud, ha permitido que las expectativas de vida de las mascotas sean mayores, de allí que la presentación de casos con cáncer haya aumentado, toda vez que la edad es un factor predisponente para las neoplasias (Moulton, 1978).

El presente estudio tuvo como objetivo determinar la frecuencia de neoplasias diagnosticadas histopatológicamente en el Laboratorio de Patología de la Facultad de Medicina Veterinaria de la Universidad Nacional Mayor de San Marcos, Lima, en el periodo comprendido entre enero de 1996 y diciembre de 2007. Se hizo un estudio retrospectivo de los casos de neoplasias en felinos en base a los registros de diagnóstico citológico e histopatológico de biopsias y necropsias de felinos. Se consideró las variables edad ( 0 a $<5,5$ a $<9, \geq 9$ años), sexo, raza (cruces, pura), órgano o tejido afectado y tipo de neoplasia según su origen celular. Se utilizó como base referencial la clasificación de tumores propuesta por Moulton (1978). Los datos se presentan en base a frecuencia e intervalos de confianza al 95\%.

El laboratorio tuvo un registro de 208 casos en felinos en el periodo 1996-2007, de los cuales 73 casos correspondieron a neoplasias felinas (35.1\%). En un estudio previo, con registros del mismo laboratorio que abarcó el periodo 1973-1992, se encontró que los casos de neoplasias correspondieron al 19.6\% de casos en felinos (Yabar, 1994). El incremento relativo en el presente estudio se puede deber a factores tales como la mayor preocupación de los dueños por saber la causa del problema de su mascota, mejora en la relación Médico Veterinario-dueño, mayor posibilidad para la realización de exámenes citológicos e histopatológicos, aumento del interés en el estudio de neoplasias, y a la mayor expectativa de vida que tienen las mascotas en la actualidad (De Vivero, 2009).

Los felinos con 9 o más años de edad presentaron la mayor frecuencia de neoplasias (49.3 $\pm 11.8 \%$ ), en tanto que la frecuencia de casos de neoplasias en felinos entre 5 y 9 años y entre 0 y 5 años fue de $23.2 \pm 10.0 \%$ y $27.5 \pm 10.5 \%$. El efecto de la edad sobre la presentación de neoplasias ha sido ampliamente demostrado (Gross et al., 1992; Delisle y Devauchelle, 2000; Morgan et al., 2004). Autores como Moulton (1978) y Ogilvie y Moore (1996) señalan que la edad más común de presentación de neoplasias se encuentra dentro del rango de 9 a 13 años.

Los animales cruzados presentaron una mayor frecuencia $(74.7 \pm 10.1 \%)$ que aquellos de razas puras. Por otro lado, las hembras fueron más afectadas $(72.9 \pm 10.4 \%)$ que los machos ( $27.1 \pm 10.4 \%)$, dado que la mayor frecuencia de neoplasias fue a nivel de la glándula mamaria (30.1 $\pm 10.7 \%)$. Gatti (2008) menciona este tipo de tumores en machos (1-2\%), a diferencia del presente estudio, donde la totalidad de neoplasias mamarias fue hallada en hembras. Asimismo, Ogilvie y Moore (1996) reportan una mayor presencia de linfoma en machos, mientras que en este trabajo la presentación del linfoma fue similar entre sexos ( 6 casos en hembras y 5 en machos).

En el Cuadro 1 se puede observar que las neoplasias más frecuentes son las de origen epitelial (53.5 $\pm 11.6 \%$ ), seguidas por las de origen conectivo y hematopoyético, mientras que las de origen endotelial, muscular y misceláneo se manifestaron con una baja frecuencia. 
Cuadro 1. Frecuencia de neoplasias en felinos, según su origen celular, observadas durante el periodo 1996-2007

\begin{tabular}{|c|c|c|c|c|c|}
\hline $\begin{array}{l}\text { Derivación } \\
\text { (origen) }\end{array}$ & N. ${ }^{\circ}$ & $\%$ & Tipo de neoplasia & N. ${ }^{\circ}$ & $\%$ \\
\hline \multirow{6}{*}{ Conectivo } & \multirow{6}{*}{12} & \multirow{6}{*}{16.9} & Fibroma & 1 & 1.4 \\
\hline & & & Fibrosarcoma & 3 & 4.2 \\
\hline & & & Mixosarcoma & 1 & 1.4 \\
\hline & & & Sarcoma & 1 & 1.4 \\
\hline & & & Lipoma & 2 & 2.8 \\
\hline & & & Mastocitoma & 1 & 1.4 \\
\hline Endotelial & 4 & 5.6 & Hemangiosarcoma & 4 & 5.6 \\
\hline Hematopoyético & 12 & 16.9 & Linfoma & 12 & 16.9 \\
\hline \multirow{2}{*}{ Muscular } & \multirow{2}{*}{4} & \multirow{2}{*}{5.6} & Leiomiosarcoma & 2 & 2.8 \\
\hline & & & Rabdomiosarcoma & 2 & 2.8 \\
\hline \multirow{7}{*}{ Epitelial } & \multirow{7}{*}{38} & \multirow{7}{*}{53.5} & Papiloma & 2 & 2.8 \\
\hline & & & Pilotrixoma & 1 & 1.4 \\
\hline & & & Adenoma & 1 & 1.4 \\
\hline & & & $\begin{array}{l}\text { Carcinoma de células } \\
\text { escamosas }\end{array}$ & 9 & 12.7 \\
\hline & & & Otros carcinomas & 3 & 4.2 \\
\hline & & & $\begin{array}{l}\text { Adenocarcinoma } \\
\text { mamario }\end{array}$ & 19 & 26.8 \\
\hline & & & Otros adenocarcinomas & 3 & 4.2 \\
\hline Misceláneos & 1 & 1.4 & Disgerminoma & 1 & 1.4 \\
\hline Total & 71 & 100.0 & & 71 & 100.0 \\
\hline
\end{tabular}

Las neoplasias epiteliales, las cuales incluyen en orden de importancia de presentación a las neoplasias de glándulas mamarias y los carcinomas de células escamosas, fueron las de mayor frecuencia. Resultados similares fueron obtenidos por Yabar (1994) y Morgan et al. (2004), aunque Birchard y Schering (1996) encontraron una mayor cantidad de neoplasias hematopoyéticas que epiteliales.
Las neoplasias de origen conectivo y hematopoyético presentaron una frecuencia de presentación similar; sin embargo, si se analiza el tipo individual de neoplasia, el linfoma fue el de mayor frecuencia (16.9\%). Esta neoplasia es considerada por diversos autores como la de mayor frecuencia en el felino (Yabar, 1994; Birchard y Schering, 1996; Couto y Nelson, 2005). 
Las neoplasias malignas fueron las más frecuentes ( $\mathrm{n}=60$ casos, $88.2 \pm 7.7 \%$ ) frente al $11.8 \%$ de neoplasias benignas, similar a lo encontrado por Moulton (1978) en un estudio realizado en 1198 felinos.

\section{Literatura Citada}

1. Birchard SJ, Sherding RG. 1996. Manual clínico de pequeñas especies. Vol 1. México: McGraw Hill-Interamericana. $947 \mathrm{p}$.

2. Couto CG Nelson RW. 2005. Medicina interna de animales pequeños. $3^{\mathrm{a}} \mathrm{ed}$. Vol 2. Buenos Aires: Inter-Médica. 1453 p.

3. Delisle F, Devauchelle P. 2000. Skin tumours dermatology. In: Guaguere E, Prelaud P (eds). A practical guide to feline dermatology. London: Merial. p 15.1-15.13.

4. De Vivero LE. 2009. Frecuencia de neoplasias caninas diagnosticadas en el Laboratorio de Histología, Embriología y Patología Veterinaria de la FMVUNMSM. Periodo 1995-2006. Tesis de Médico Veterinario. Lima: Facultad de Medicina Veterinaria, Univ Nacional Mayor de San Marcos. 63 p.
5. Gatti RM. 2008. Neoplasia de mama en un felino macho. En: Temas de medicina felina. Anuario 2008: Buenos Aires: Asociación Argentina de Medicina Felina. p 63-64.

6. Gross TL, Ihrke PJ, Walder EJ. 1992. A macroscopic and microscopic evaluation of canine and feline skin disease. In: Veterinary dermatophatology. St. Louis: Ed Mosby. p 520.

7. Martínez EM, Pérez C. 2007. Situación actual de la oncología de pequeños animales en España. [Internet], [15 enero 2010]. Disponible en: http:// www.colvema.org/PDF/Oncologia.pdf

8. Morgan, RV, Ronald MB, Swartout $M M . ~ 2004$. Clínica de pequeños animales. $4^{\mathrm{a}}$ ed. España: Elsevier. 1355 p.

9. Moulton JE. 1978. Tumors in domestic animals. $2^{\text {nd }}$ ed. USA: University of California. 469 p.

10. Ogilvie GK, Moore AS. 1996. Managing the veterinary cancer patient. Veterinary Learning Systems. $542 \mathrm{p}$.

11. Yabar RL. 1994. Tipificación histopatológica de las neoplasias en gatos. Periodo 1973-1992. Tesis de Médico Veterinario. Lima: Facultad de Medicina Veterinaria, Univ Nacional Mayor de San Marcos. 24 p. 